\title{
$16 S$ rDNA analysis of the intestinal microbes in osteoporotic rats
}

\author{
Yan SUN ${ }^{1,2}$, Hui-Jie ZHANG ${ }^{2}$, Ran $\mathrm{CHEN}^{4}$, Hong-Bin ZHAO ${ }^{3 *}$ and Wen-Hui LEE ${ }^{2 *}$ \\ ${ }^{1}$ Pharmaceutical College \& Key Laboratory of Pharmacology for Natural Products of Yunnan Province, Kunming Medical University, \\ Kunming, Yunnan 650500, China \\ ${ }^{2}$ Key Laboratory of Bio-active Peptides of Yunnan Province/Key Laboratory of Animal Models and Human Disease Mechanisms of the \\ Chinese Academy of Sciences, Kunming Institute of Zoology, Kunming, Yunnan 650032, China \\ ${ }^{3}$ Department of Emergency Trauma, The First People's Hospital of Yunnan Province, Affiliated Hospital of Kunming University of \\ Science and Technology, Kunming, Yunnan 650032, China \\ ${ }^{4}$ Department of Clinical Lab, The Second Affiliated Hospital of Kunming Medical University, Kunming, Yunnan 650000, China
}

Received September 7, 2020; Accepted February 12, 2021; Published online in J-STAGE February 23, 2021

\begin{abstract}
This study aimed to reveal the differences in intestinal microbes in osteoporotic rats. The rats were divided into two groups: the control and OP (osteoporosis) groups $(n=6)$. Days 0 and 70 were set as the time points. The rats in the OP group underwent bilateral ovariectomy $(\mathrm{OVX})$. Differences between the control and OP groups were determined by 16S rDNA analysis. The relative abundances of OTUs and alpha/beta diversities were determined at days 0 days and 70. The abundances of Verrucomicrobia at the phylum level and Aerococcus, Coprobacillus, Veillonella, Anaerobiospirillum, Flavobacterium, Comamonadaceae, Ohtaekwangia, etc., at the genus level were found to be different between the control_70d and OP 70d groups. KEGG ontology analysis showed that the function of lipid metabolism could be related to OP. The 16S rDNA analysis in the OP rats revealed that intestinal microbes take part in the processes of $O P$ and could affect lipid metabolism. Further study of the relationship between $\mathrm{OP}$ and intestinal microbes is necessary, and the prospect for intestinal microbes is a potential treatment for OP.
\end{abstract}

Key words: 16S rDNA analysis, osteoporosis, intestinal microbe

\section{INTRODUCTION}

The 16S rDNA gene is the gene encoding primitive nuclear ribosomes [1]. Its sequence consists of 9 variable regions and 10 conserved areas. The conserved zone sequence reflects the relationship between species, while the variable zone sequence can vary among existing species. The relevant data reveals the species classification, the abundance of species, and the evolution of the system. For intestinal microbes, representative operational taxonomic units (OTUs) can be obtained by PCR amplification of an extracted sample and 16S rDNA sequencing [2]. An OTU corresponds to a $16 \mathrm{~S}$ rDNA sequence and a unique bacterium [3]. Through OTU analysis, microbial diversity and relative abundances can be determined.

More than 100 trillion microbes are present in the human intestine [4]. The main constituents of the intestinal microbes include Firmicutes, Bacteroidetes, Proteobacteria, Verrucomicrobia, Fusobacteria, and Actinobacteria, as well as others [5]. These intestinal microbes participate in many physiological processes and metabolisms [6], and they are key factors in the balance of human health.

Osteoporosis (OP) is characterized by low bone mass, bone microstructure deterioration, bone fragility, and even brittle fracture susceptibility [7]. Approximately 200 million people suffer from osteoporosis worldwide [8,9]. OP has a high morbidity and mortality rate, resulting in a massive social and economic burden $[10,11]$. For healthy people, bone absorption and resorption are strictly regulated to maintain sufficient bone mass, structural and mechanical properties. This balance is disrupted as OP progresses [12]. It has been reported that the absorption of nutriments and probiotics could regulate bone health through changes in intestinal microbes [13-15].

In this study, an OP model was reproduced, and intestinal microbes were analyzed by $16 \mathrm{~S}$ rDNA sequencing. Alpha/beta diversity indices, phylum and genus comparisons, and gene function predictions were used to study the relationship between $\mathrm{OP}$ and the intestinal microbes. One or more microbes could be related to OP. This study aimed to determine the changes in intestinal microbes and provide evidence for studying and treating OP.

*Corresponding authors. Hong-Bin Zhao and Wen-Hui Lee (E-mails: 596829191@qq.com; leewh@mail.kiz.ac.cn) (Supplementary material: refer to PMC https://www.ncbi.nlm.nih.gov/pmc/journals/2480/) 


\section{MATERIALS AND METHODS}

\section{Animals}

All animal experiments were approved by the Animal Study Committee of Kunming Medical University (No. KMMU 2015007) and were conducted according to the requirements of the NIH Guidelines for the Care and Use of Laboratory Animals. A total of 12 three-month-old female SD rats ( $270 \pm 15 \mathrm{~g}$; Dossy Co., China) were maintained under standard conditions with a controlled temperature $\left(21-23^{\circ} \mathrm{C}\right)$ and a strict $12 \mathrm{hr}$ light/dark cycle. All rats were fed standard rat chow and allowed free access to distilled water ad libitum at all times during the acclimatization and experimental treatment periods.

\section{OP model}

After 7 days of adaptation, the rats were randomly divided into a bilateral ovariectomy (OVX) group and sham-surgery group. Briefly, 6 rats underwent bilateral laparotomy (sham rats), and 6 rats were subjected to bilateral OVX. The animals were administered an intraperitoneal injection of $30 \mathrm{mg} / \mathrm{kg}$ sodium pentobarbital (Servicebio Co., China) to induce deep anesthesia. All animals were fasted for $12 \mathrm{hr}$ before surgery. Benzylpenicillin sodium (60,000 IU/kg; Harbin Pharmaceutical Co., China) was administered for three consecutive days after operations.

\section{BMD analysis}

After surgery, the whole-body bone mineral density (BMD) of each rat was measured using dual-energy X-ray absorptiometry (DXA; Lunar Prodigy Advance, GE Medical Systems LUNAR, Madison, WI) every week. Specific software for small animals (enCORE2004 software, version 8.80.001, GE Medical Systems) was used.

\section{Grouping}

The control group was comprised of sham rats, and the OP group was comprised of OVX rats, with six rats in each group. Each rat was marked with a digital ear tag.

\section{Sample collection}

The day after reproduction of the OP model was considered the start point. On the first (day 0) and last (day 70) days, intestinal contents were collected from each rat in the control and OP groups for follow-up 16S rDNA analysis of microbes, resulting in a total 24 samples being collected (samples C_0d_16, C_70d_1-6, OP_0d_1-6, and OP_70d_1-6). All samples were placed in sterile $\mathrm{PBS}$, stored at $-70^{\circ} \mathrm{C}$, and transported on dry ice to BGI-Kunming (BGI Co., China).

\section{$16 S$ rDNA amplicon sequencing}

DNA was extracted from the samples, and the V3-V4 region of the 16S rDNA genes was amplified by polymerase chain reaction (PCR) with a universal forward primer and a unique barcoded fusion reverse primer (341F, ACTCCTACGGGAGGCAGCAG; 806R, GGACTACHVGGGTWTCTAAT). PCR was performed using the following conditions: 3 min of denaturation at $94^{\circ} \mathrm{C}$; 25 cycles of denaturation at $94^{\circ} \mathrm{C}$ for $45 \mathrm{sec}$, annealing at $50^{\circ} \mathrm{C}$ for $60 \mathrm{sec}$, and elongation at $72^{\circ} \mathrm{C}$ for $90 \mathrm{sec}$; and final extension at $72^{\circ} \mathrm{C}$ for $10 \mathrm{~min}$. The amplicons were purified using AMPure beads (Axygen). Barcoded libraries were generated by emulsion PCR and sequenced in a V5 to V4 reverse direction on a 318 chip using the $400 \mathrm{bp}$ sequencing kit of an Ion Torrent Personal Genome Machine (PGM) system, according to the manufacturer's instructions [16]. The output sequences of each sample were no less than 50,000 pairs corresponding to 25,000 clean targets, and informatics methods (strategies: PE101/PE150/PE250/PE300) were applied. The clean targets were clustered by USEARCH v7.0.1090 as OTUs.

\section{Data analysis}

Figures for comparisons of $16 \mathrm{~S}$ rDNA data were generated with the GraphPad Prism 8.0, STAMP, LEfSe, and R packages (including QIIME2, ggplot, etc.). KEGG functions were analyzed by PORCUSt and divided into three KEGG orthology (KO) levels. Statistical significance was determined by non-parametric test for group comparisons (IBM SPSS Statistics 21.0, IBM Corp., Armonk, NY, USA), and $\mathrm{p}<0.05$ indicated statistical significance.

\section{RESULTS}

\section{$16 S \mathrm{rDNA} O \mathrm{OTU}$ results for the OP rats}

After 23 weeks, the rats in a bilateral OVX and sham groups were examined by DXA. The BMD of the OVX group was 0.177 $\pm 0.006 \mathrm{~g} / \mathrm{cm}^{3}$, and that of the sham group was $0.188 \pm 0.008 \mathrm{~g} /$ $\mathrm{cm}^{3}(\mathrm{p}<0.05)$. Reproduction of the OP model was successful.

The intestinal microbes on days 0 and 70 are shown in Fig. 1A; the significant difference OTU numbers were OTU_4, OTU_7, and OTU_10. The numbers of OTUs in the groups according to timepoint (C_0d, C_70d, OP_0d, and OP 70d) were 567, 536, 560 , and 598 ( $>>0.05)$, respectively (Fig. 1B). The differences in OTUs for all samples in each group are shown in Fig. 1C, and there were obvious individual differences. The numbers of core-Pan OTU plots in the control group decreased from day 0 to day 70 and the individual differences of rats increased. The number of core-Pan OTU plots in the OP group increased from 162 to 213 after 70 days.

\section{The alpha- and beta-diversity analysis results for the $O P$ rat}

The Ace, Chao, and Shannon coverage values were greater than $98 \%$, and there were smooth rarefaction curves (Fig. 2A). Comparison of the three indices between the control and OP groups revealed that the Ace, Chao, and Shannon indices in the OP group were increased on day $70(p>0.05)$. Comparison of the three indices of the OP group between day 0 and day 70 revealed that the Ace and Chao indices increased from day 0 to day $70(\mathrm{p}>0.05)$; the Shannon index decreased from day 0 to day $70(p>0.05)$, and the standard deviations of the OP samples were large at day 70 (Fig. 2B).

A Bray-Curtis analysis based on the quantitative characteristics of different species in the samples showed differences in each sample (Fig. 2C). The differences between samples are shown by color depth. The separation rates of PC1, PC2, and PC3 were $33.7 \%, 25.4 \%$, and $10.5 \%$, respectively (Fig. 2D). There were obvious differences between the $\mathrm{C}_{-} 70 \mathrm{~d}$ and $\mathrm{OP} 770 \mathrm{~d}$ groups via principal coordinate analysis (PCoA) analysis, and the values of PCoA1 and PCoA2 were $25.46 \%$ and $12.94 \%$ (Fig. 2E). The resulting cladogram was analyzed by the least discriminant analysis (LDA) method, and the changes in intestinal microbes in each group are shown in Fig. 2F. Differences in Fibrobacteres, Planctomycetia, Spirochaetes, and other bacteria were observed between the control group and OP group from day 0 to day 70 . 

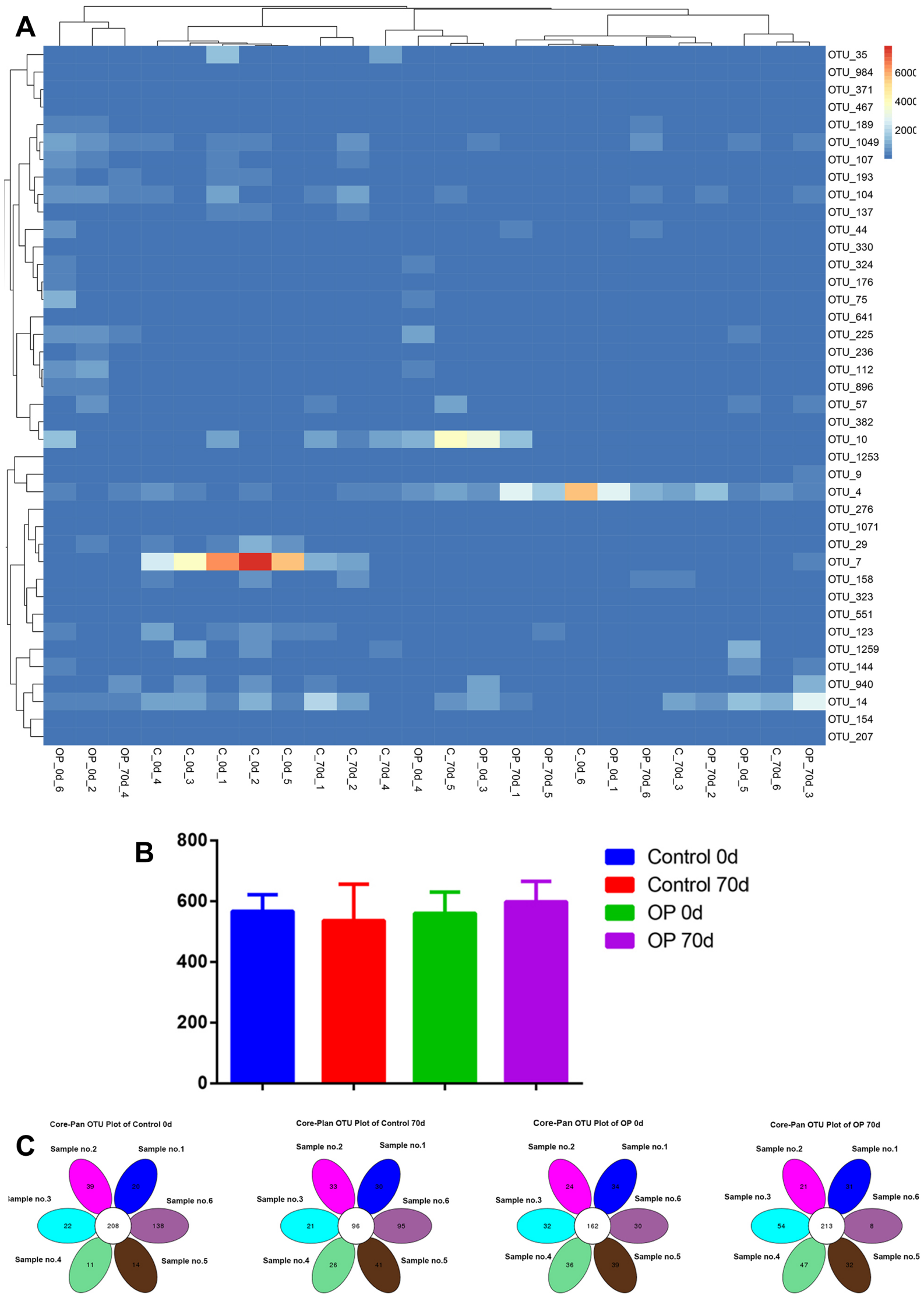

Fig. 1. The 16S rDNA OTU results of the OP rats. (A) The OTU heatmap for the samples. The names on the right are OTU IDs, and the color value of each square in each row indicates the relative abundance of the OTUs. (B) The OTU numbers for each group. The values are presented as the mean \pm standard deviation (SD). (C) The OTU differences in each sample. The white color indicates the abundance of the common OTUs for each sample, and the other colors indicate the unique abundance of the OTUs to each sample. 
A

C_0d: $98.18 \% \pm 2.38 \%$ C_70d: $98.25 \% \pm 2.40 \%$ OP_0d: $98.17 \% \pm 2.50 \%$ OP_70d: $98.05 \% \pm 2.57 \%$
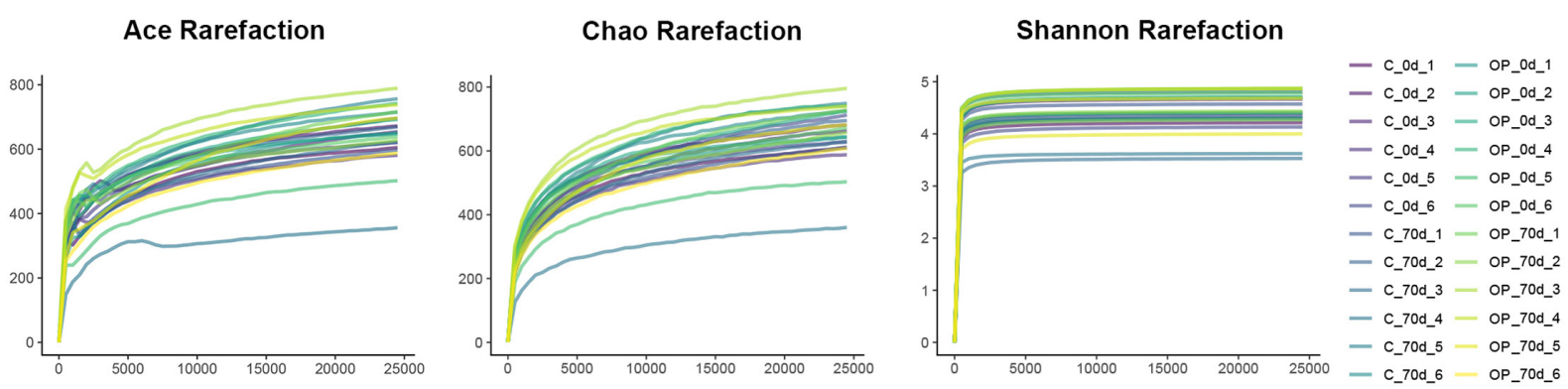

B

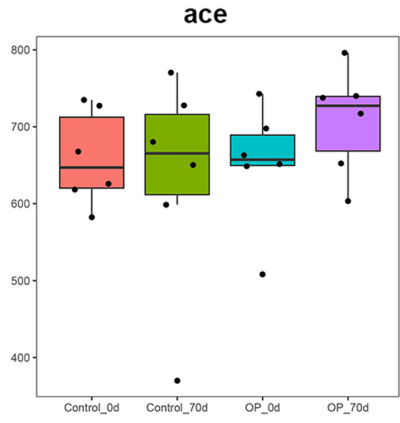

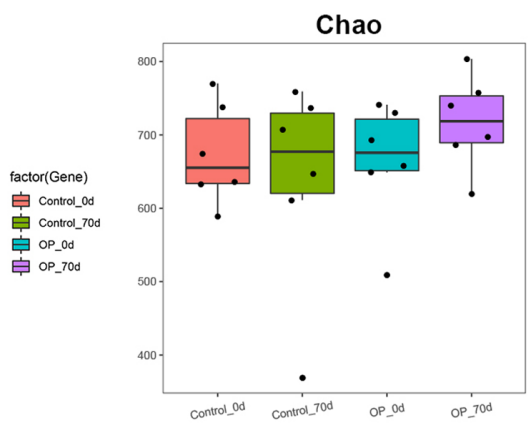
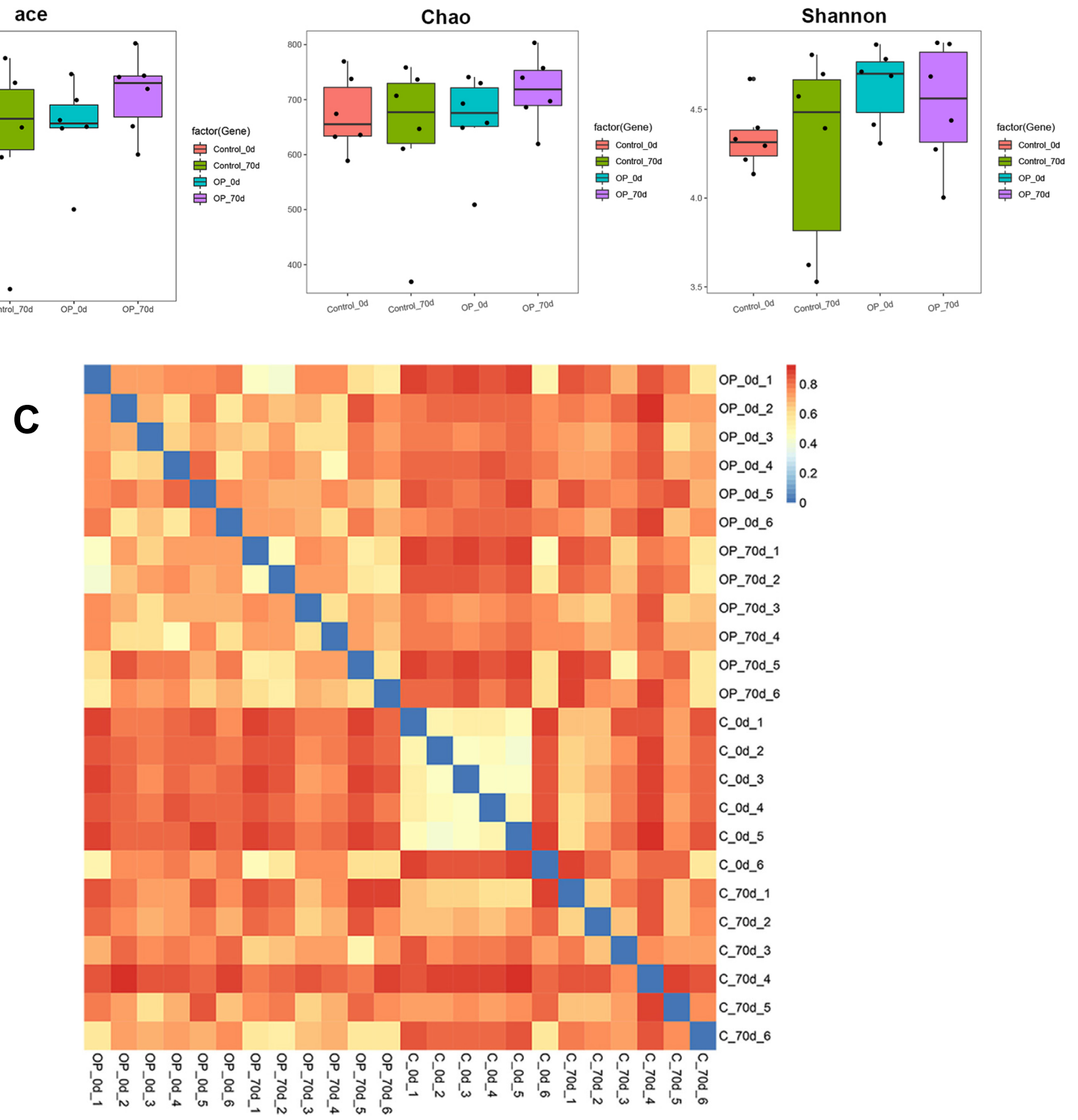

Fig. 2. The alpha- and beta-diversity analysis results of the OP rats. (A) The coverage values for all samples in each group. The number of sequences is indicated by the $\mathrm{x}$-axis, and the number of OTUs is indicated by the $\mathrm{y}$-axis. The percentages for each group represent the correlation between the sequencing results and the actual microbes in the samples. (B) The Ace, Chao, and Shannon indices for each group. (C) The heatmap of Bray-Curtis diversity. Differences are indicated by different colors on the coordinate axis. (D) The PCA results of each group. PC1, PC2, and PC3 represent the first, second, and third principal components, respectively. Each plot represents a sample, and the same color indicates the same group. (E) The PCoA results of each group. Each point corresponds to a different sample colored by compartment, and each group is indicated by a different color. The percentage of variance indicated in each axis corresponds to the fraction of the total variance explained by the projection. (F) The LDA effect size taxonomic cladogram comparing all samples. Different colors indicate species with significant differences between groups, and the logarithmic LDA score is set to 2 . The magnitude of the plot is proportional to the relative abundance. 
D PCA c.od c.70d $\triangle$ op.od op.70d
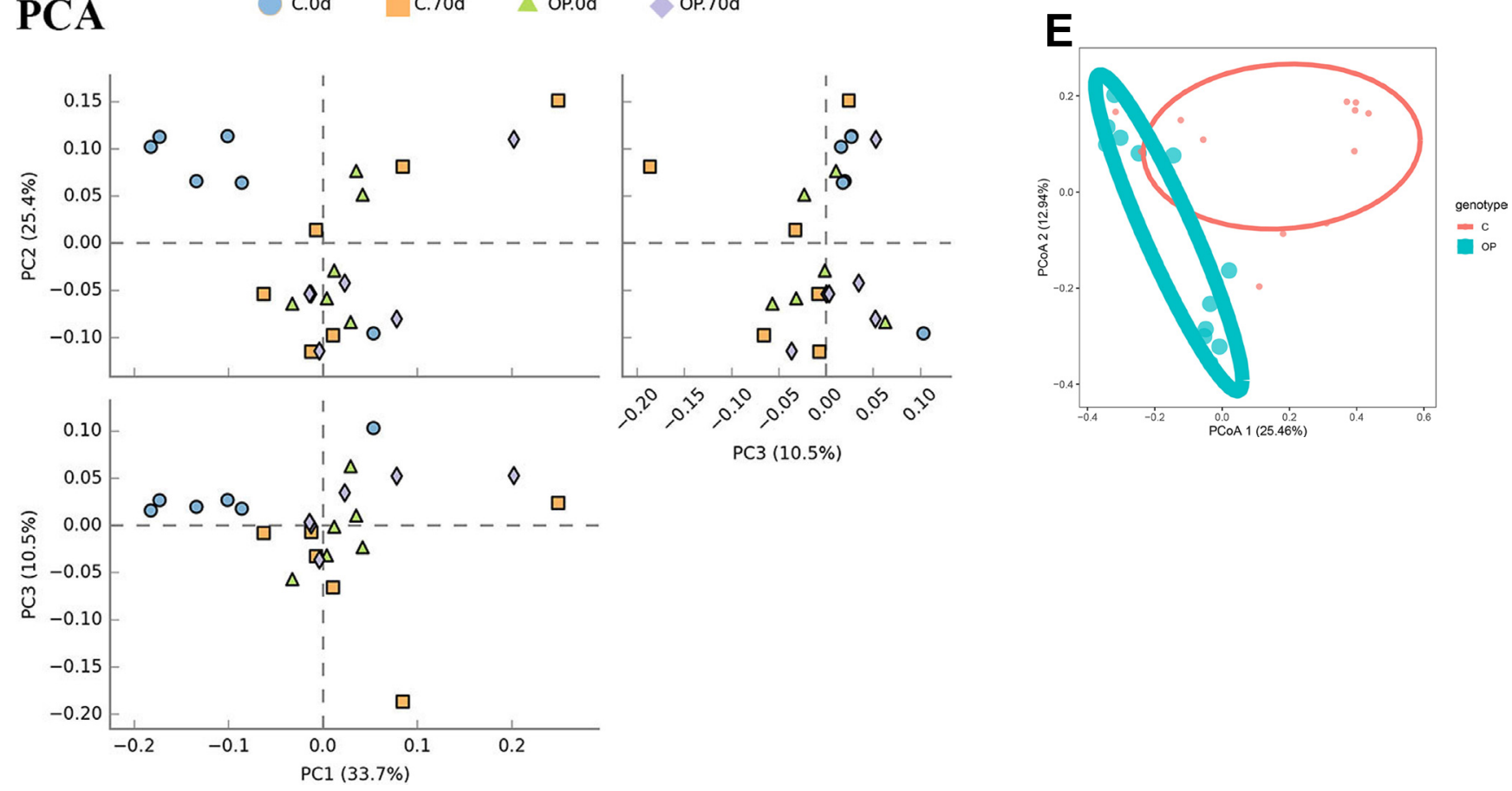

$\mathbf{F}$

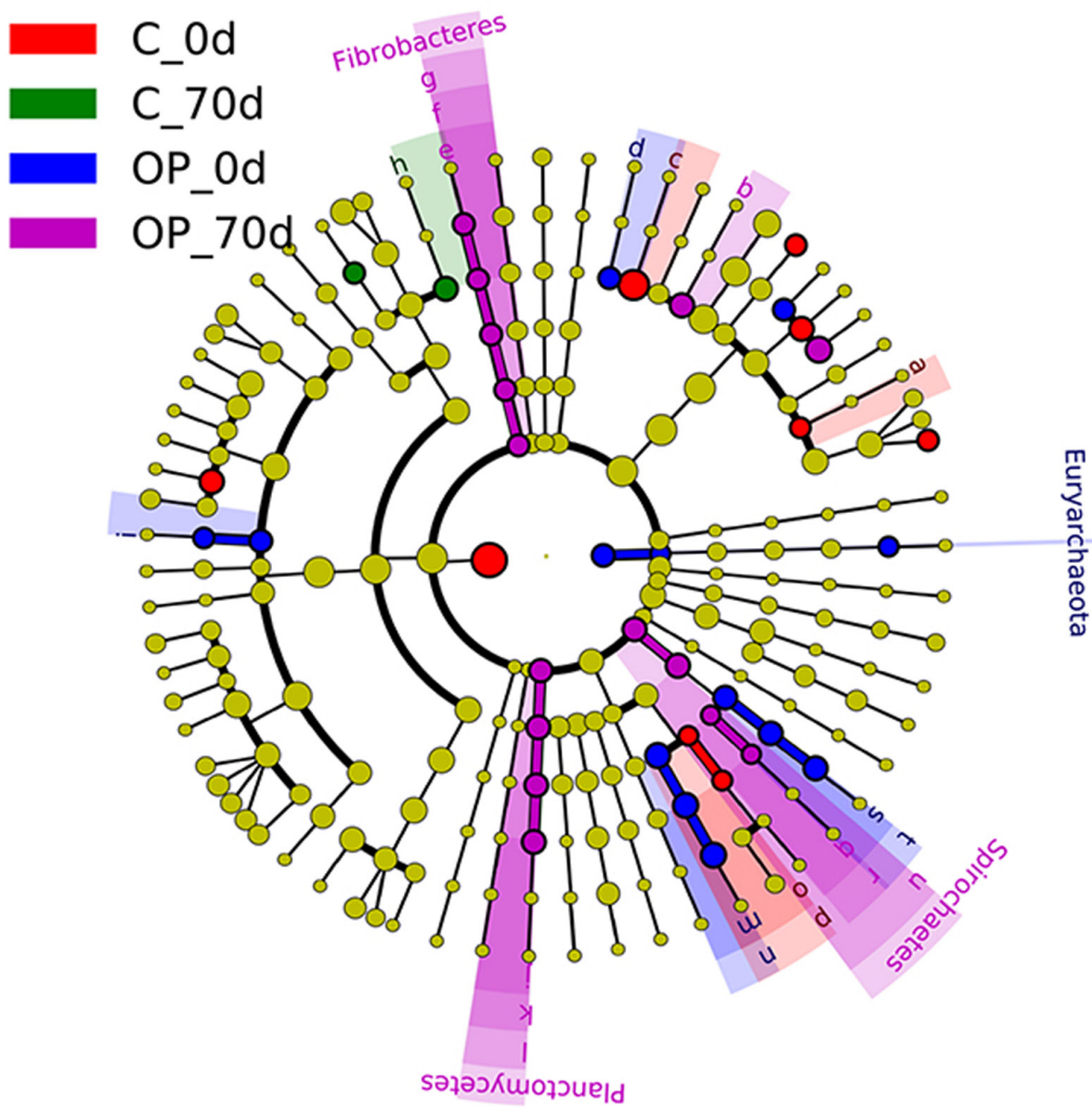

a: Barnesiellaceae

b: RF16

c: S24_7

d: p_2534_18B5

e: Fibrobacteraceae

f: Fibrobacterales

g: Fibrobacteria

h: Aerococcaceae

i: Mogibacteriaceae

j: Pirellulaceae

k: Pirellulales

I: Planctomycetia

$\mathrm{m}$ : Succinivibrionacé̃

$\mathrm{n}$ : Aeromonadales

o: Enterobacteriacea

p: Enterobacteriales

q: Sphaerochaetacea

r: Sphaerochaetales

s: Spirochaetaceae

t: Spirochaetales

u: Spirochaetes

Fig. 2. 


\section{Compositions and changes at the phylum and genus levels}

The compositions and changes at the phylum and genus levels are shown in Fig. 3A and C. At the phylum level, comparison of $\mathrm{C}_{-} 70 \mathrm{~d}$ and OP_70d revealed that the significantly different microbe constituent was Verrucomicrobia ( $p<0.05$, Fig. 3B). At the genus level, comparison of C_ $70 \mathrm{~d}$ and OP_70d revealed that the top four significantly different microbe constituents were Aerococcus, Coprobacillus, Veillonella, and Anaerobiospirillum $(\mathrm{p}<0.05$, Fig. 3D). The most differentially abundant genus between C_70d and OP_70d was Ohtaekwangia $(\mathrm{p}<0.05$, Fig. $3 \mathrm{E})$. The differences in intestinal microbes in comparisons within groups (day 0 vs day 70) are shown in the supplementary material (Supplementary Fig. 1) An evolution tree was established, showing microbial variations, OTU IDs, phylum and genus taxonomy, and other information (Fig. 3F).

\section{KO level function analysis of the OP rats}

The functions of intestinal microbes were based on the KEGG database and divided into three KO levels. It was determined that the intestinal microbes in the control and OP groups had KO functions at level 1, level 2, and level 3, which represented their functional classifications (Fig. 4A-C). The functions related to OP could be cell growth and lipid metabolism at level 2 and lipid biosynthesis protein at level 3. Compared with the control group on day 70 , the OP group on day 70 exhibited a higher enrichment of lipid metabolism (C_70d, 60.34\% $\pm 6.27 \%$; OP_70d, 61.71\% $\pm 6.67 \%$ ).

\section{DISCUSSION}

The study of intestinal microbes has been focused on using antibiotic or probiotic administration and fecal transplantation for treating diseases [17]. 16S rDNA sequencing is an effective method for studying intestinal microbes. A DXA method was used to evaluate BMD in this study, and an OP model was successfully reproduced, as there was a statistically significant difference in BMD between the groups. This study reproduced OP rats at 23 weeks after OVX surgery [18], and intestinal contents were collected on days 0 and 70 . In a previous study with estrogen deficiency induced by ovariectomy, Escherichia coli increased without the inhibition of estrogen [19], and it is known that intestinal microbes have an influence on maintenance of the balance of the bone microenvironment [20]. The sequencing results in the present study revealed the changes in the intestinal microbes in the OP group from day 0 to day 70 . These microbes could be related to the process of OP and could become a potential treatment for OP in the future.

Before the alpha-diversity analysis, the coverage values were calculated as mean \pm standard deviation (SD) values. In general, the coverage values for all samples were greater than $98 \%$, and the $16 \mathrm{~S}$ rDNA sequencing was good. The extended terminal $\mathrm{x}$-axis was the number of sequences of the sample. If the curve tends to be flat, the sequencing depth is sufficient to compare samples. However, if the number of sequencing reads increased, it is still possible to find additional OTUs. Conversely, if the curve is not saturated and additional OTUs could be found, then the 16S rDNA sequencing is not good. The alpha-diversity indices were tested by the Wilcoxon test, and the significant differences under different conditions were selected. Ace, an alpha-diversity index, was used to estimate the OTU index in the community, as described by Pan et al. [21]. The difference between Ace and Chao is that they use different algorithms. The greater the Ace and Chao values are, the greater the total number of species. The Shannon index is used to estimate the microbial diversity in samples. The larger the Shannon values are, the higher the diversity of the communities. At day 0 , these indices were similar in the control and OP groups. On day 70, although there were no significant differences in alpha-diversity indices between the control and OP groups, the values were increased. Compared with the values in the control group, the Ace and Chao values and the Shannon values were higher and lower, respectively, in the OP group. There were more species and fewer communities in the OP group than in the control group.

PCA (principal component analysis) is a visual method for studying data similarities or differences [22]. The differences between samples and groups were determined by sorting the characteristics of the eigenvalues and eigenvectors and selecting the main characteristics. PCoA is similar to PCA but uses a different coordinate system [23]. Differences between the control and OP groups were observed by both PCA and PCoA. The closer the distance between two plots is, the more similar the differences. A taxonomic cladogram was obtained by LDA comparing all samples [24]. The PCA results only present the data for the samples, whereas the LDA results shows the relationship between species and the feature choice, which was tested by the Wilcoxon test and linear discriminant test. In addition, LDA can sort functional characteristics according to values, and LDA identifies mainly significant differences of species in groups. There were significant differences in PC1, PC2, and PC 3 of the samples. The OP and control groups had obvious differences according to PCoA. The LDA cladogram shows the different species and relative abundances. The top four significant species were Fibrobacteres, Euryarchaeota, Spirochaetes, and Planctomycetia.

Fibrobacteres was identified in 1988 at the genus level and isolated from the cow rumen/rat cecum. It contains xylanases but lacks the genes necessary to metabolize xylan degradation products for energy transduction [25, 26]. Some studies have reported that Euryarchaeota is a marine archaea, and Euryarchaeota exists in the human intestinal tract and oral cavity and is related to methanogenic archaea [27-29]. Spirochaetes, found in humans [30], is related to hydrogenotrophic methanogens [31]. Planctomycetia has an asymmetric bilayer outer membrane and takes part in the digestion in seaweed grazers [32]. The above evidence suggests that there are relationships between Fibrobacteres, Euryarchaeota, Spirochaetes, Planctomycetia, and OP.

At the phylum level, the significantly different microbe constituent was Verrucomicrobia. At the genus level, the top four significantly different microbe constituents were Aerococcus, Coprobacillus, Veillonella, and Anaerobiospirillum.

Verrucomicrobia can be detected in soil and water [33, 34] and is suggested to degrade glycolate and polysaccharides [35, 36]. Aerococcus is a fastidious gram-positive coccus that is most frequently associated with urinary tract infections (UTIs), bacteremia, and endocarditis [37, 38]. Coprobacillus was first isolated in 1999 and has highly variable sugar fermentation patterns [39]. Veillonella is a gram-negative anaerobic coccus that resists tetracycline and penicillin [40]. Anaerobiospirillum can cause bacteremia, septicemia, and pyomyositis [41]. In truth, 

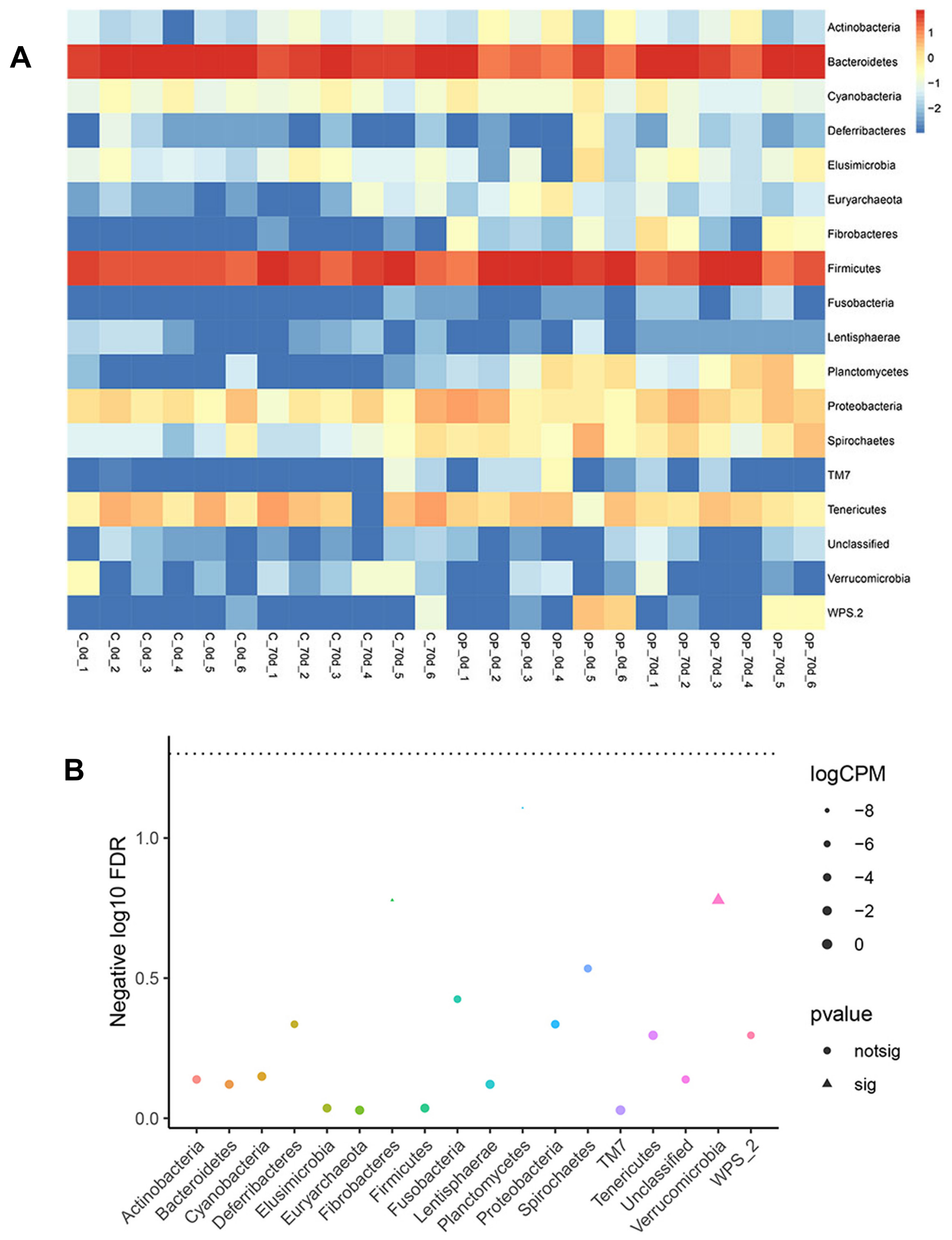

Fig. 3. Compositions and changes at the phylum and genus levels. (A) The phylum heatmap for the samples. (B) The relative abundances of the phyla. The phyla are indicated by the $\mathrm{x}$-axis, and the $\log _{10}$ FDR is indicated by the $\mathrm{y}$-axis. $\log _{\mathrm{cpm}}$ means $\log _{2} \mathrm{CPM}$. Levels are presented as $\mathrm{P}$ values between the control_70d and OP_70d groups, and $\mathrm{p}<0.05$ indicates significance. (C) The genus heatmap for the samples. (D) The relative abundances of the genera. The genera are indicated by the $\mathrm{x}$-axis, and the $\log _{10}$ FDR is indicated by the y-axis. $\log _{\mathrm{cpm}}$ means $\log _{2} \mathrm{CPM}$. Levels are presented as $P$ values between the control_70d and OP_70d groups, and $\mathrm{p}<0.05$ indicates significance. (E) Comparison of genera among the C_0d, C_70d, and OP_70d groups. (F) Evolution tree. The ruler indicates the variation in the gene, and the color indicates the phylum. The circle indicates the genus and OTU ID. 


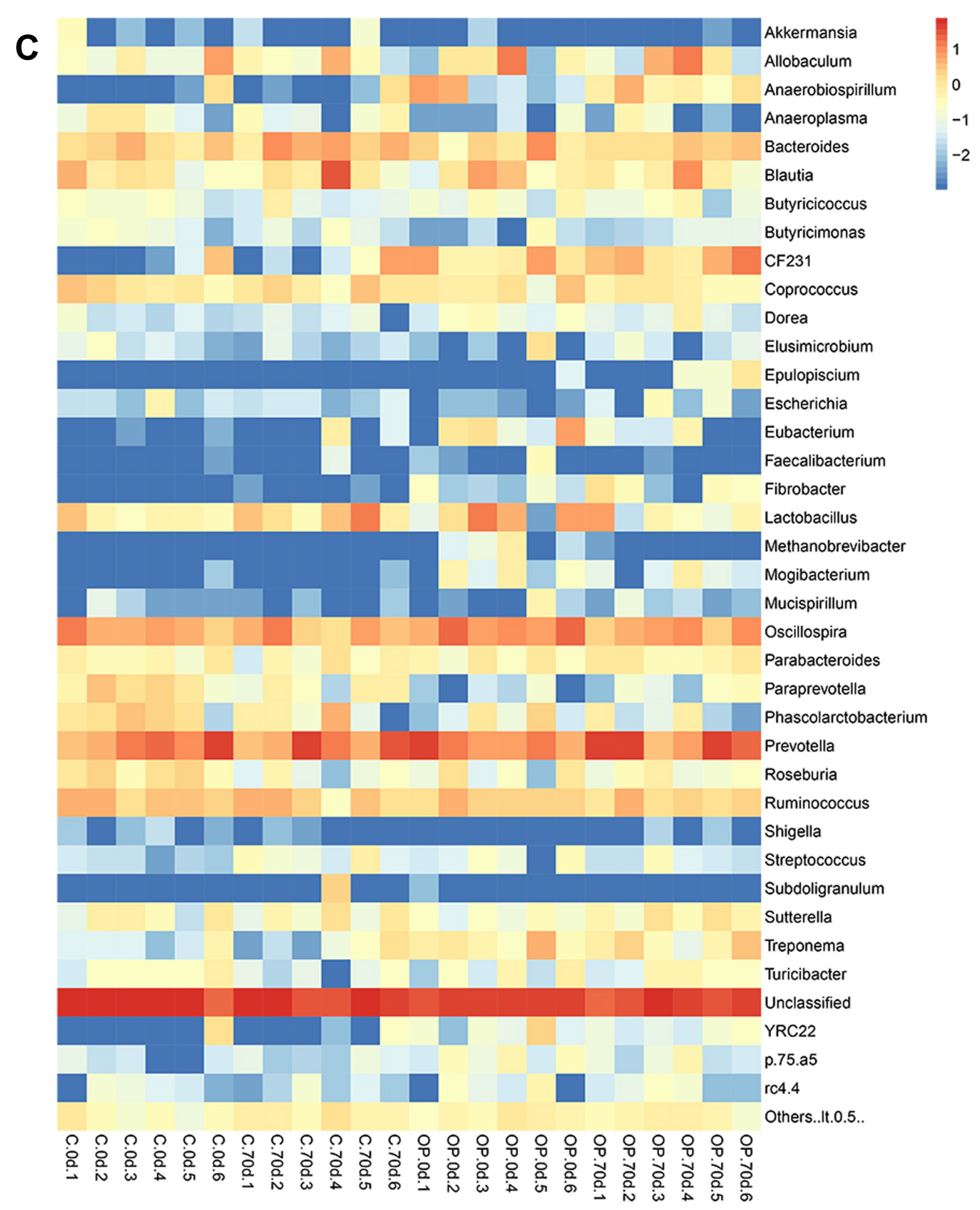

D

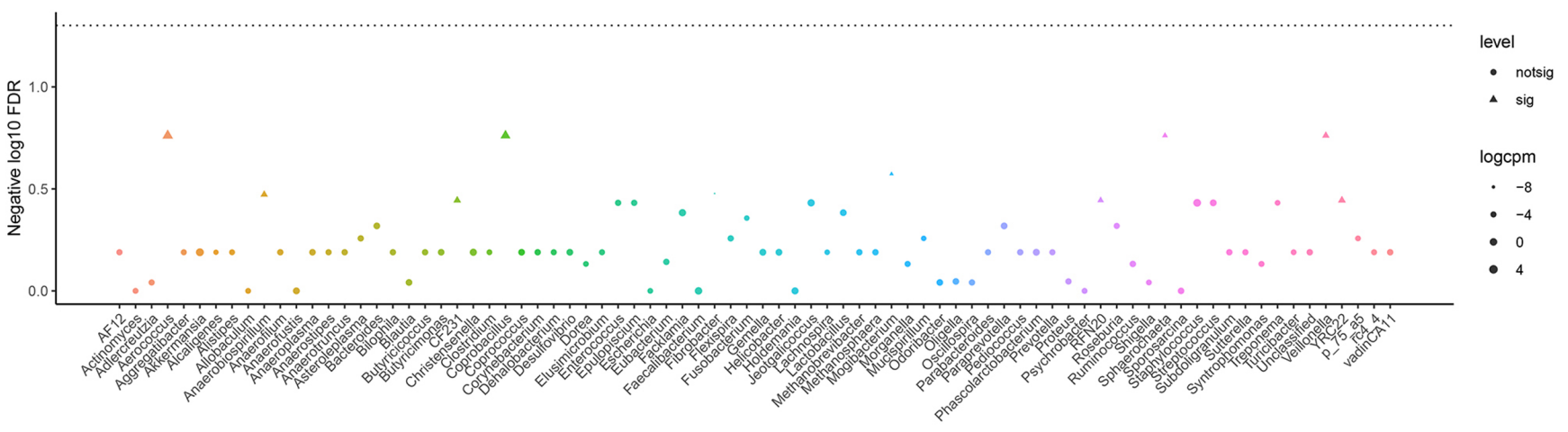

Fig. 3. 
E

Contrpl 70d

OP $70 d$
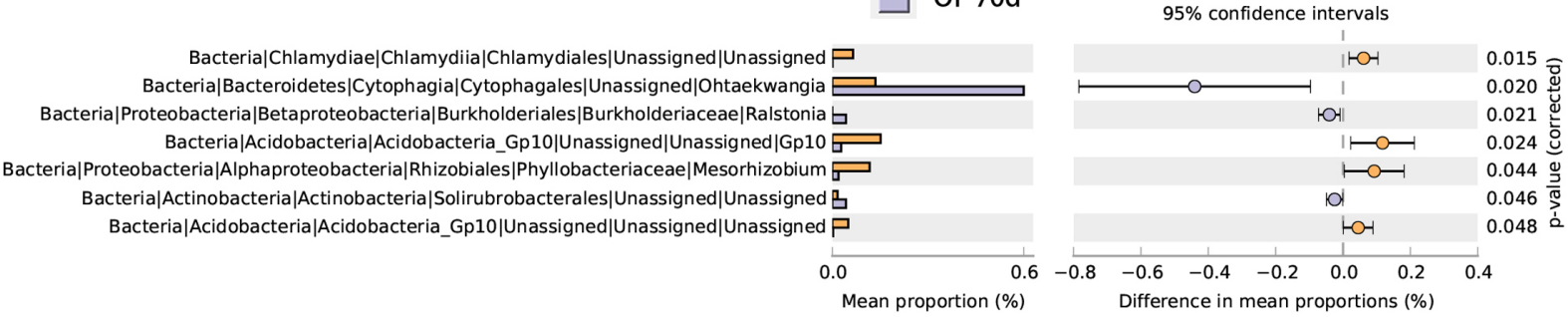

Tree scale: 0.1

\section{$\mathbf{F}$}

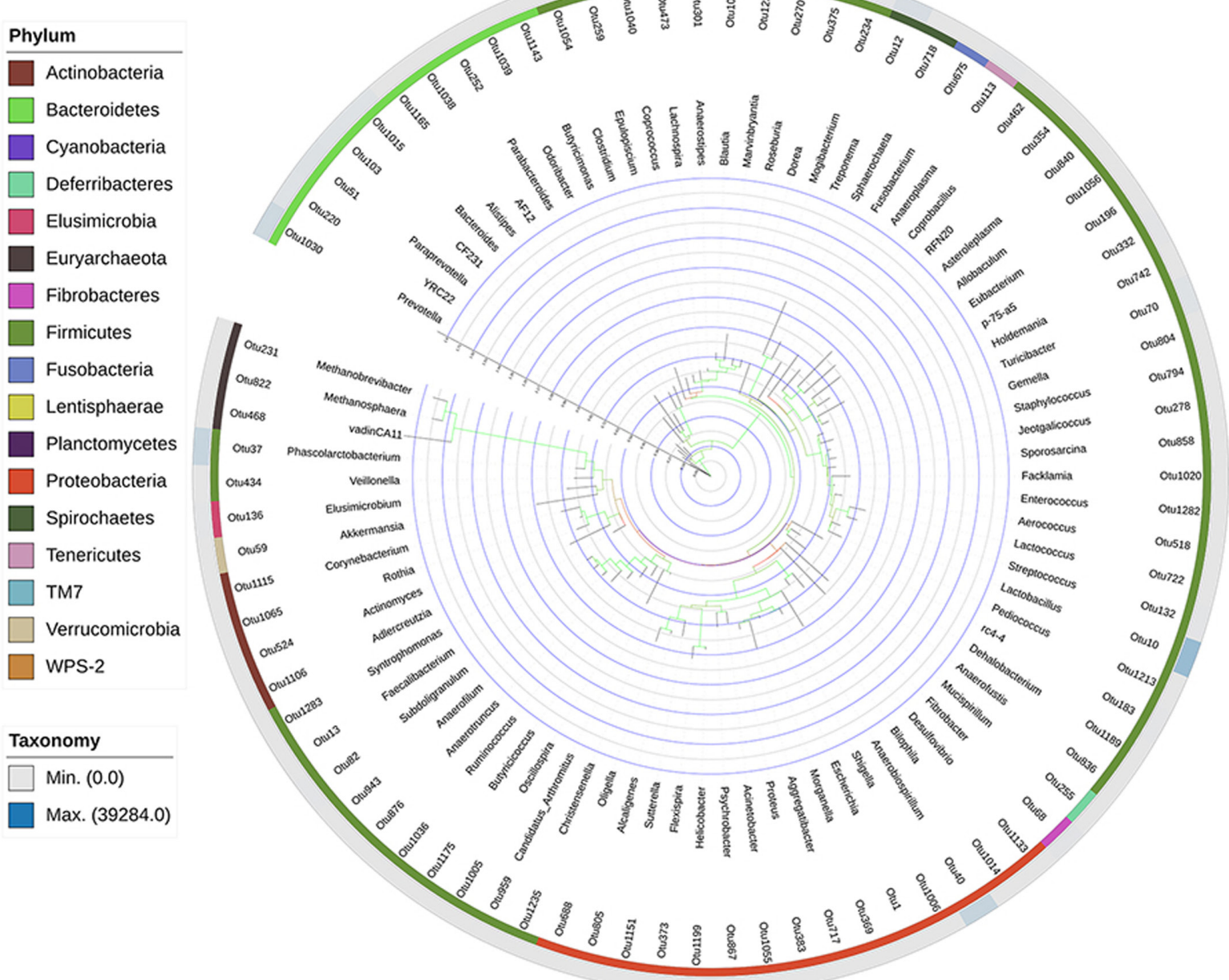

Fig. 3.

studies of these microbes have been poor, and there are few explanations for diseases. In addition to the microbes mentioned above, these findings indicate that intestinal microbes could take part in the process of OP. Meanwhile, a number of the bacteria identified in the present study were environmental microbes and archaea. This study cannot explain the relationship between intestinal microbes and OP, and further study could be interesting.

The combination of IQ-Tree (http://www.iqtree.org/doc/Home) analysis with OTU IDs and annotation of samples facilitates the identification of dominant strains. In the present study, it showed the evolutionary relationships and relative abundances of samples $[42,43]$. KO analysis showed that the gene functions of the intestinal microbe constituents in the control and OP groups had gene functions. These results revealed that lipid metabolism and lipid biosynthesis proteins could be related to OP disease. It has been reported that hyperlipidemia adversely affects bone growth and BMD. Oxidized lipids may subsequently induce bone loss by inhibiting the differentiation of bone-forming osteoblasts and promoting the differentiation of bone-resorbing osteoclasts [44]. BMD and low-density lipoprotein (LDL) cholesterol levels are inversely correlated in mice and rats. The mechanism of OP is related to lipid metabolism and lipid peroxidation [45]. 


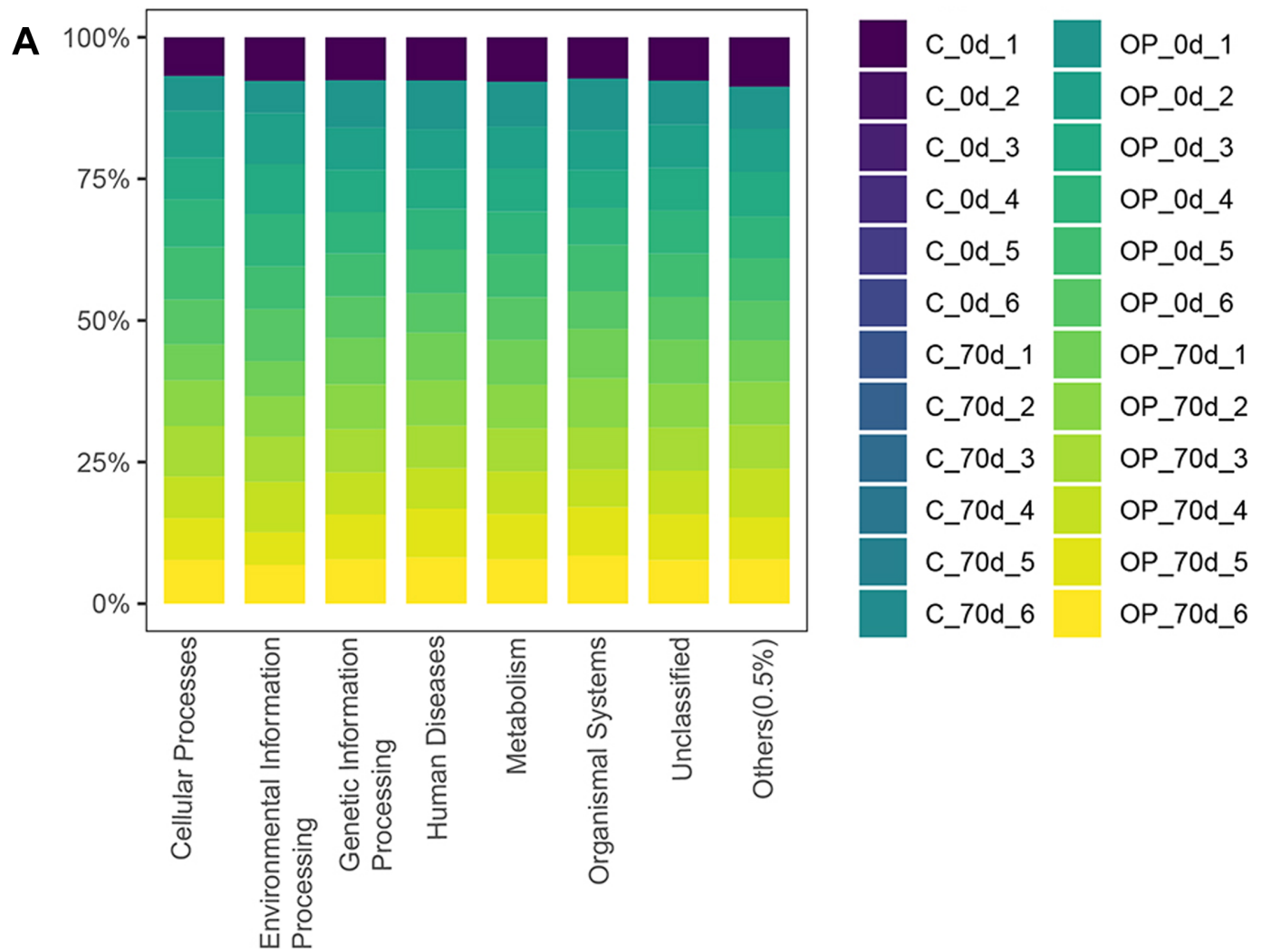

B

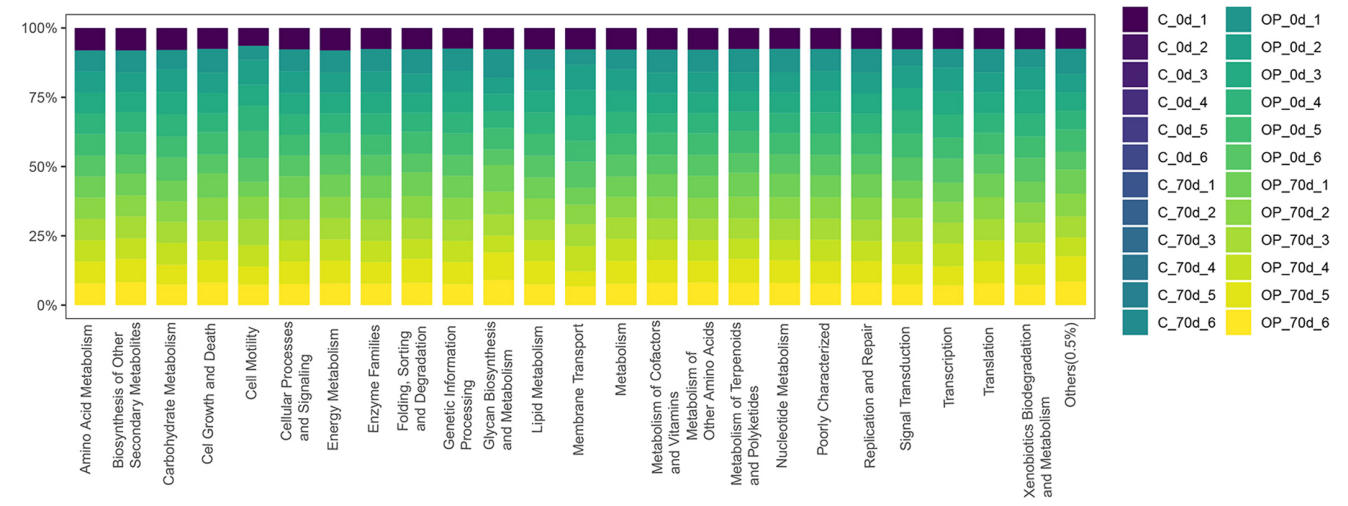

C

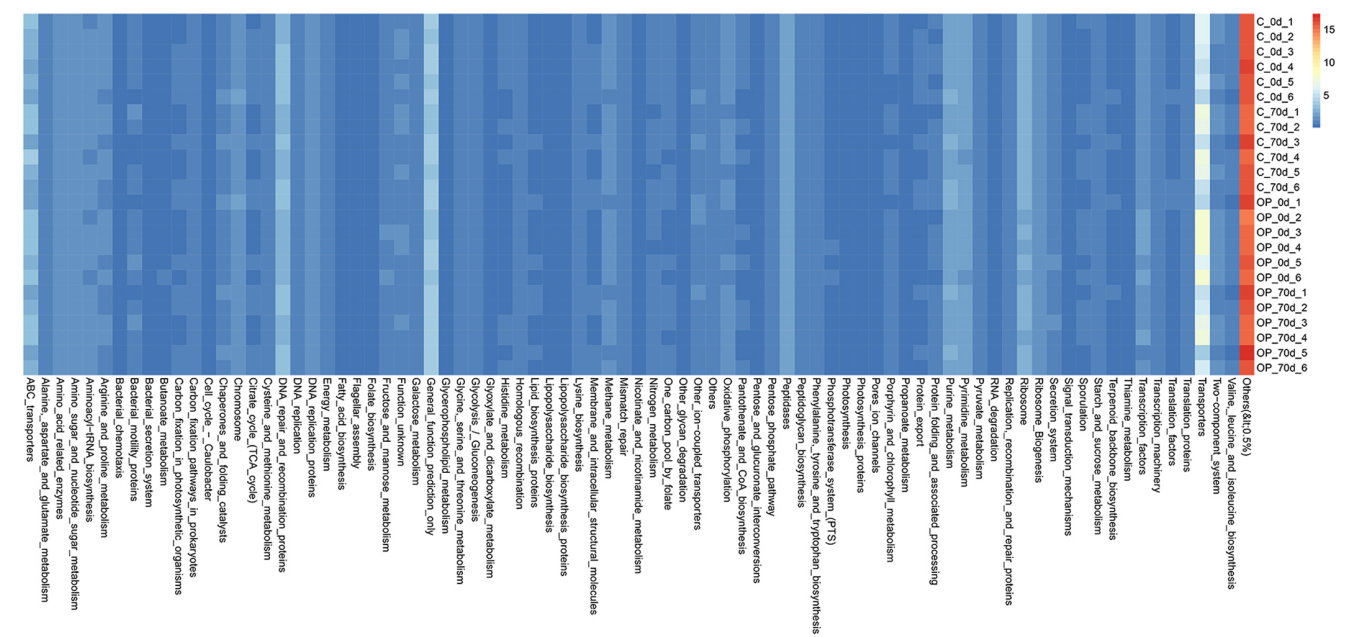

Fig. 4. KO function analysis. The percentages of the colors indicate the differences in the relative abundances for each group. (A) The results of KO analysis at level 1 . The gene name is indicated by the $\mathrm{x}$-axis, and the relative abundance is indicated by the $\mathrm{y}$-axis. (B) The results of KO analysis at level 2. The gene name is indicated by the $\mathrm{x}$-axis, and the relative abundance is indicated by the y-axis. (C) The results of KO analysis at level 3. 
The changes in OP could be focused on functions of intestinal microbes related to lipid metabolism.

To date, over 1,000 distinct bacterial species have been recovered from the human intestine. These bacterial species in humans are called intestinal microbes. The intestinal microbes regulate physiological and biochemical processes [46], and it has been reported that Euphorbia prostrata inhibits osteoclasts and increases osteoblasts. E. prostrata regulates bone absorption and resorption by regulating Lactobacillus and Lactococcus [47]. Firmicutes and Bacteroidetes can regulate obesityassociated gut dysbiosis. Ophiopogonin D alters the FirmicutesBacteroidetes ratio in obese-prone rats $[48,49]$. 16S rDNA sequencing revealed a portion of the intestinal microbes related to lipid metabolism, and the relative abundances in the OP group were higher than in the control group. The improvement of lipid metabolism could promote the proliferation and differentiation of osteoblasts and inhibit the activity of osteoclasts [50]. Further studies focused on the intestinal microbes related to lipid metabolism after 70 days would be a potential prospect for the direction of future research. In the present study, the OP group exhibited enrichment of other functions that were not directly related to OP disease, and their mechanisms could be interesting for treating OP.

This study suggested that several microbes were changed in OP rats and that their functions were related to lipid metabolism. However, there are obvious differences between rats and humans, including differences in diet, bone architecture, and microbiome. No evidence in this study could explain how the intestinal microbes affected OP, and a limitation of this study was that the investigations were not sufficiently deep.

$16 \mathrm{~S}$ rDNA analysis revealed the differences of intestinal microbes in rats and that the function of lipid metabolism could affect OP. Comparison of the C_70d and OP 70d groups revealed differences in the intestinal microbes, especially in Aerococcus, Coprobacillus, Veillonella, Anaerobiospirillum, Flavobacterium, Comamonadaceae, Ohtaekwangia, and other genera. Further study is necessary, and the prospect for intestinal microbes is a potential treatment for OP.

\section{AUTHORS' CONTRIBUTIONS}

HBZ and WHL designed this study. YS and HJZ completed the experiments. YS and RC analyzed the data. YS drafted the manuscript.

\section{FUNDING}

This study was supported by National Science Foundation of China united fund U1502227.

\section{CONFLICT OF INTEREST}

To the best of our knowledge, the named authors, including Yan Sun, Hui-Jie Zhang, Ran Chen, Hong-Bin Zhao, and Wen-Hui Lee, have no conflicts of interest, financial or otherwise.

\section{REFERENCES}

1. Both B, Krupp G, Stackebrandt E. 1991. Direct sequencing of double-stranded polymerase chain reaction-amplified 16S rDNA. Anal Biochem 199: 216-218. [Medline] [CrossRef]
2. Drancourt M, Bollet C, Carlioz A, Martelin R, Gayral JP, Raoult D. 2000. 16S ribosomal DNA sequence analysis of a large collection of environmental and clinical unidentifiable bacterial isolates. J Clin Microbiol 38: 3623-3630. [Medline] [CrossRef]

3. Piotrowska A, Gosiewski T, Bulanda M, Brzychczy-Wloch M. 2016. Using of the $16 \mathrm{~S}$ rDNA sequencing for identification of Lactobacillus species. Med Dosw Mikrobiol 68: 5-11. [Medline]

4. Samuel BS, Shaito A, Motoike T, Rey FE, Backhed F, Manchester JK, Hammer RE, Williams SC, Crowley J, Yanagisawa M, Gordon JI. 2008. Effects of the gut microbiota on host adiposity are modulated by the short-chain fatty-acid binding $\mathrm{G}$ protein-coupled receptor, Gpr41. Proc Natl Acad Sci USA 105: 16767-16772. [Medline] [CrossRef]

5. Ley RE, Bäckhed F, Turnbaugh P, Lozupone CA, Knight RD, Gordon JI. 2005. Obesity alters gut microbial ecology. Proc Natl Acad Sci USA 102: 11070-11075. [Medline] [CrossRef]

6. Arumugam M, Raes J, Pelletier E, Le Paslier D, Yamada T, Mende DR, Fernandes GR, Tap J, Bruls T, Batto JM, Bertalan M, Borruel N, Casellas F, Fernandez L, Gautier L, Hansen T, Hattori M, Hayashi T, Kleerebezem M, Kurokawa K, Leclerc M, Levenez F, Manichanh C, Nielsen HB, Nielsen T, Pons N, Poulain J, Qin J, Sicheritz-Ponten T, Tims S, Torrents D, Ugarte E, Zoetendal EG, Wang J, Guarner F, Pedersen O, de Vos WM, Brunak S, Doré J, Antolín M, Artiguenave F, Blottiere HM, Almeida M, Brechot C, Cara C, Chervaux C, Cultrone A, Delorme C, Denariaz G, Dervyn R, Foerstner KU, Friss C, van de Guchte M, Guedon E, Haimet F, Huber W, van Hylckama-Vlieg J, Jamet A, Juste C, Kaci G, Knol J, Lakhdari O, Layec S, Le Roux K, Maguin E, Mérieux A, Melo Minardi R, M'rini C, Muller J, Oozeer R, Parkhill J, Renault P, Rescigno M, Sanchez N, Sunagawa S, Torrejon A, Turner K, Vandemeulebrouck G, Varela E, Winogradsky Y, Zeller G, Weissenbach J, Ehrlich SD, Bork P, MetaHIT Consortium. 2011. Enterotypes of the human gut microbiome. Nature 473: 174-180. [Medline] [CrossRef]

7. Sietsema DL. 2020. Fighting the epidemic: bone health and osteoporosis. Nurs Clin North Am 55: 193-202. [Medline] [CrossRef]

8. Miro-Blanch J, Yanes O. 2019. Epigenetic regulation at the interplay between gut microbiota and host metabolism. Front Genet 10: 638. [Medline] [CrossRef]

9. Johnell O, Kanis JA, Oden A, Johansson H, De Laet C, Delmas P, Eisman JA, Fujiwara S, Kroger H, Mellstrom D, Meunier PJ, Melton LJ 3rd, O’Neill T, Pols H, Reeve J, Silman A, Tenenhouse A. 2005. Predictive value of BMD for hip and other fractures. J Bone Miner Res 20: 1185-1194. [Medline] [CrossRef]

10. Park C, Jang S, Lee A, Kim HY, Lee YB, Kim TY, Ha YC. 2015. Incidence and mortality after proximal humerus fractures over 50 years of age in South Korea: national claim data from 2008 to 2012. J Bone Metab 22: 17-21. [Medline] [CrossRef]

11. Ström O, Borgström F, Kanis JA, Compston J, Cooper C, McCloskey EV, Jönsson B. 2011. Osteoporosis: burden, health care provision and opportunities in the EU: a report prepared in collaboration with the International Osteoporosis Foundation (IOF) and the European Federation of Pharmaceutical Industry Associations (EFPIA). Arch Osteoporos 6: 59-155. [Medline] [CrossRef]

12. Kanis JA, Cooper C, Rizzoli R, Reginster JY, Scientific Advisory Board of the European Society for Clinical and Economic Aspects of Osteoporosis (ESCEO) and the Committees of Scientific Advisors and National Societies of the International Osteoporosis Foundation (IOF). 2019. European guidance for the diagnosis and management of osteoporosis in postmenopausal women. Osteoporos Int 30: 3-44. [Medline] [CrossRef]

13. Bliuc D, Nguyen ND, Milch VE, Nguyen TV, Eisman JA, Center JR. 2009. Mortality risk associated with low-trauma osteoporotic fracture and subsequent fracture in men and women. JAMA 301: 513-521. [Medline] [CrossRef]

14. Hippisley-Cox J, Coupland C. 2009. Predicting risk of osteoporotic fracture in men and women in England and Wales: prospective derivation and validation of QFractureScores. BMJ 339: b4229. [Medline] [CrossRef]

15. Lorentzon M, Cummings SR. 2015. Osteoporosis: the evolution of a diagnosis. J Intern Med 277: 650-661. [Medline] [CrossRef]

16. Chen C, Song X, Wei W, Zhong H, Dai J, Lan Z, Li F, Yu X, Feng Q, Wang Z, Xie H, Chen X, Zeng C, Wen B, Zeng L, Du H, Tang H, Xu C, Xia Y, Xia H, Yang H, Wang J, Wang J, Madsen L, Brix S, Kristiansen K, Xu X, Li J, Wu R, Jia H. 2017. The microbiota continuum along the female reproductive tract and its relation to uterinerelated diseases. Nat Commun 8: 875. [Medline] [CrossRef]

17. Roman P, Cardona D, Sempere L, Carvajal F. 2019. Microbiota and organophosphates. Neurotoxicology 75: 200-208. [Medline] [CrossRef]

18. Sun Y, Chen R, Zhu D, Shen ZQ, Zhao HB, Lee WH. 2020. Osteoking improves OP rat by enhancing HSP90- $\beta$ expression. Int J Mol Med 45: 1543-1553. [Medline]

19. Yang Y, Qu C, Liang S, Wang G, Han H, Chen N, Wang X, Luo Z, Zhong C, Chen Y, Li L, Wu W. 2018. Estrogen inhibits the overgrowth of Escherichia coli in the rat intestine under simulated microgravity. Mol Med Rep 17: 2313-2320. [Medline]

20. Collins FL, Rios-Arce ND, Schepper JD, Parameswaran N, McCabe LR. 2017. The potential of probiotics as a therapy for osteoporosis. Microbiol Spectr 5. [Medline]

21. Pan HY, Chao A, Foissner W. 2009. A nonparametric lower bound for the number of species shared by multiple communities. J Agric Biol Environ Stat 14: 452-468. [Medline] [CrossRef]

22. Ringnér M. 2008. What is principal component analysis? Nat Biotechnol 26: 303-304. [Medline] [CrossRef] 
23. Bookstein FL. 2017. A method of factor analysis for shape coordinates. Am J Phys Anthropol 164: 221-245. [Medline] [CrossRef]

24. Zhou Y, Zhang B, Li G, Tong T, Wan X. 2017. GD-RDA: a new regularized discriminant analysis for high-dimensional data. J Comput Biol 24: 1099-1111. [Medline] [CrossRef]

25. Montgomery L, Fu YK. 1988. Detection of cellulose-binding proteins in electrophoresis gels by filter paper affinity blotting. Anal Biochem 174: 204-208. [Medline] [CrossRef]

26. Abdul Rahman N, Parks DH, Vanwonterghem I, Morrison M, Tyson GW, Hugenholtz P. 2016. A phylogenomic analysis of the bacterial phylum fibrobacteres. Front Microbiol 6: 1469. [Medline] [CrossRef]

27. Friedrich MW, Schmitt-Wagner D, Lueders T, Brune A. 2001. Axial differences in community structure of Crenarchaeota and Euryarchaeota in the highly compartmentalized gut of the soil-feeding termite Cubitermes orthognathus. Appl Environ Microbiol 67: 4880-4890. [Medline] [CrossRef]

28. Horz HP, Conrads G. 2010. The discussion goes on: what is the role of Euryarchaeota in humansbArchaea 2010: 967271.

29. Haro-Moreno JM, Rodriguez-Valera F, López-García P, Moreira D, Martin-Cuadrado AB. 2017. New insights into marine group III Euryarchaeota, from dark to light. ISME J 11: 1102-1117. [Medline] [CrossRef]

30. John GD. 1914. Some researches on Spirochetes occurring in the alimentary tract of man and some of the lower animals. Marcus Beck Laboratory Reports: 48-70.

31. Lee SH, Park JH, Kim SH, Yu BJ, Yoon JJ, Park HD. 2015. Evidence of syntrophic acetate oxidation by Spirochaetes during anaerobic methane production. Bioresour Technol 190: 543-549. [Medline] [CrossRef]

32. Aires T, Serebryakova A, Viard F, Serrão EA, Engelen AH. 2018. Acidification increases abundances of Vibrionales and Planctomycetia associated to a seaweedgrazer system: potential consequences for disease and prey digestion efficiency. PeerJ 6: e4377. [Medline] [CrossRef]

33. He S, Stevens SLR, Chan LK, Bertilsson S, Glavina Del Rio T, Tringe SG, Malmstrom RR, McMahon KD. 2017. Ecophysiology of freshwater Verrucomicrobia inferred from metagenome-assembled genomes. MSphere 2: e00277-17. [Medline] [CrossRef]

34. O'Farrell KA, Janssen PH. 1999. Detection of Verrucomicrobia in a pasture soil by PCR-mediated amplification of 16S rRNA genes. Appl Environ Microbiol 65: 4280-4284. [Medline] [CrossRef]

35. Martinez-Garcia M, Brazel DM, Swan BK, Arnosti C, Chain PS, Reitenga KG, Xie G, Poulton NJ, Lluesma Gomez M, Masland DE, Thompson B, Bellows WK, Ziervogel K, Lo CC, Ahmed S, Gleasner CD, Detter CJ, Stepanauskas R. 2012. Capturing single cell genomes of active polysaccharide degraders: an unexpected contribution of Verrucomicrobia. PLoS One 7: e35314. [Medline] [CrossRef]

36. Paver SF, Kent AD. 2010. Temporal patterns in glycolate-utilizing bacterial community composition correlate with phytoplankton population dynamics in humic lakes. Microb Ecol 60: 406-418. [Medline] [CrossRef]

37. Humphries RM, Lee C, Hindler JA. 2011. Aerococcus urinae and trimethoprimsulfamethoxazole. J Clin Microbiol 49: 3934-3935. [Medline] [CrossRef]

38. Adomavicius D, Bock M, Vahl CF, Siegel E. 2018. Aerococcus urinae mitral valve endocarditis-related stroke: a case report and literature review. J Investig Med High Impact Case Rep 6: 2324709618758351. [Medline]

39. Kageyama A, Benno Y. 2000. Coprobacillus catenaformis gen. nov., sp. nov., a new genus and species isolated from human feces. Microbiol Immunol 44: 23-28. [Medline] [CrossRef]

40. Reig M, Mir N, Baquero F. 1997. Penicillin resistance in Veillonella. Antimicrob Agents Chemother 41: 1210. [Medline] [CrossRef]

41. Ready D, Pratten J, Roberts AP, Bedi R, Mullany P, Wilson M. 2006. Potential role of Veillonella spp. as a reservoir of transferable tetracycline resistance in the oral cavity. Antimicrob Agents Chemother 50: 2866-2868. [Medline] [CrossRef]

42. Vázquez-Baeza Y, Pirrung M, Gonzalez A, Knight R. 2013. EMPeror: a tool for visualizing high-throughput microbial community data. Gigascience 2: 16. [Medline] [CrossRef]

43. Langille MG, Zaneveld J, Caporaso JG, McDonald D, Knights D, Reyes JA, Clemente JC, Burkepile DE, Vega Thurber RL, Knight R, Beiko RG, Huttenhower C. 2013. Predictive functional profiling of microbial communities using 16S rRNA marker gene sequences. Nat Biotechnol 31: 814-821. [Medline] [CrossRef]

44. Liu Y, Almeida M, Weinstein RS, O'Brien CA, Manolagas SC, Jilka RL. 2016. Skeletal inflammation and attenuation of Wnt signaling, Wnt ligand expression, and bone formation in atherosclerotic ApoE-null mice. Am J Physiol Endocrinol Metab 310: E762-E773. [Medline] [CrossRef]

45. Manolagas SC. 2018. The quest for osteoporosis mechanisms and rational therapies: how far we've come, how much further we need to go. J Bone Miner Res 33: 371-385. [Medline] [CrossRef]

46. Dzutsev A, Badger JH, Perez-Chanona E, Roy S, Salcedo R, Smith CK, Trinchieri G. 2017. Microbes and cancer. Annu Rev Immunol 35: 199-228. [Medline] [CrossRef]

47. Zhao X, Ai J, Mao H, Gao X. 2019. Effects of Eclipta prostrata on gut microbiota of SAMP6 mice with osteoporosis. J Med Microbiol 68: 402-416. [Medline] [CrossRef]

48. Chen S, Li X, Liu L, Liu C, Han X. 2018. Ophiopogonin D alleviates high-fat dietinduced metabolic syndrome and changes the structure of gut microbiota in mice. FASEB J 32: 1139-1153. [Medline] [CrossRef]

49. Ballini A, Scacco S, Boccellino M, Santacroce L, Arrigoni R. 2020. Microbiota and obesity: where are we now? Biology (Basel) 9: E415. [Medline]

50. Kushwaha P, Wolfgang MJ, Riddle RC. 2018. Fatty acid metabolism by the osteoblast. Bone 115: 8-14. [Medline] [CrossRef] 